\title{
Heavy-tailed Distributions and Risk Management of Equity Market Tail Events
}

\author{
Zi-Yi Guo ${ }^{1}$
}

\begin{abstract}
Traditional econometric modelling typically follows the idea that market returns follow a normal distribution. However, the concept of tail risk indicates that the distribution of returns is not normal, but skewed and has heavy tails. Thus, a heavy-tailed distribution, which accurately estimates the tail risk, would significantly improve quantitative risk management practice. In this paper, we compare four widely used heavy-tailed distributions using the S\&P 500 daily returns. Our results indicate that the Skewed $t$ distribution in Hansen (1994) has the superior empirical performance compared with the Student's $t$ distribution, the normal reciprocal inverse Gaussian distribution and the generalized hyperbolic distribution. We further showed the Skewed $t$ distribution could generate the VaR estimates closest to the nonparametric historical VaR estimates compared with other heavy-tailed distributions.
\end{abstract}

JEL Classification numbers: C46; C58; G10

Keywords: Tail risk; Value at Risk; Goodness of fit.

\section{Introduction}

The aftermath of the recent Financial Crisis emphasized the shortcomings of conventional financial wisdom. Even when everything is well crafted or normal, unexpected events can still pose a threat. These potentially rare events highlight the ongoing relevance of heavy tails throughout the finance industry. By definition, a heavy tail is a probability distribution which predicts movements of three or more standard deviations more frequently than a normal distribution. Even before the financial crisis, periods of financial stress had resulted in market conditions represented by heavy tails. This is important because normal distributions understate asset prices, stock returns and subsequent risk management strategies.

${ }^{1}$ Corporate Model Risk Management Group, Wells Fargo Bank, N.A

Article Info: Received: March 26, 2017. Revised : April 22, 2017

Published online : July 1, 2017 
As early as 1963, Mandelbrot recognized the heavy-tailed and highly peaked nature of certain financial time series. Since that time many models have been proposed to model heavy-tailed returns of financial assets (Cont, 2001). The implication that returns of financial assets have a heavy-tailed distribution may be profound to a risk manager in a financial institution. For example, $3 \sigma$ events may occur with a much larger probability when the return distribution is heavy-tailed than when it is normal. Quantile based measures of risk, such as Value at Risk, may also be drastically different if calculated for a heavy-tailed distribution. This is especially true for the highest quantiles of the distribution associated with very rare but very damaging adverse market movements.

In this paper, we discuss several widely-used heavy-tailed distributions, fit them in the Standard \& Poor's 500 index returns, and further compare their empirical performance by goodness of fit and estimation of risk measures. Our risk metric focuses the Value at Risk ( $\mathrm{VaR})$, one of the most widely used measures of market risk, credit risk or operational risk since its introduction by J.P. Morgan in 1994 (Duffie and Pan, 1998). Our results indicate the Skewed $t$ distribution has superior empirical performance compared several other widely used heavy-tailed distributions, such as the Student's $t$ distribution, the normal reciprocal inverse Gaussian distribution (NRIG) and the generalized hyperbolic distribution $(\mathrm{GH})$. We further illustrate their implications in VaR calculations.

\section{Literature Review}

It is of great importance for those in charge of managing risk to understand how financial asset returns are distributed. Since the 1960 s, empirical evidence has led in favor of various heavy tailed distributions. In a heavy-tailed distribution the likelihood that one encounters significant deviations from the mean is much greater than in the case of the normal distribution. It is now commonly accepted that financial asset returns are, in fact, heavy-tailed, and consequently, many heavy-tailed distributions have been introduced to the literature (see Rradley and Taqqu, 2003, for a survey). In addition to the standard Student's $t$ distribution, there are several other examples. For instance, Hansen (1994) introduced a type of Skewed $t$ distribution and applied it to the U.S. Dollar/Swiss Franc exchange rate. Zhu and Galbraith (2012) considered a generalized asymmetric Student's $t$ distribution with applications in financial econometrics. Barndorff-Nielsen (1977) introduced generalized hyperbolic distributions into the equity market. Socgnia and Wilcox (2014) compared various subclasses of the generalized hyperbolic distribution, including hyperbolic, variance gamma, normal inverse Gaussian (NIG) and Skewed $t$, for the daily log-returns of seven of the most liquid mining stocks listed on the Johannesburg Stocks Exchange. Barndorff-Nielsen (1997) investigated the NIG distributions, a subclass of the generalized hyperbolic distribution, in stochastic volatility modeling. Finally, Figueroa-Lopez, et al. (2011) surveyed estimation of the NIG distribution and variance gamma models for high frequency financial data.

All the above heavy-tailed distributions have already been applied into risk management area for VaR estimations. For instance, Dokov, et al. (2008) defined the Skewed $t$ distribution as a location-scale normal mixture, developed analytical formulas and numerical approximations for its $\mathrm{VaR}$ and average $\mathrm{VaR}$ and tested the results numerically. Venter and de Jongh (2002) compared the NIG distribution with the extreme value theory 
(EVT) and Student's $t$ based distributions. Venter and de Jongh found when VAR is the risk measure; the NIG based approach is more robust than the EVT method for samples of sizes up to 250 and also in larger samples if the NIG distribution fits, while the EVT method should only be used in large samples if the NIG distribution does not fit adequately. In the case of symmetric distributions, the Student's $t$ based approach compares well with the NIG based approach. However, when expected shortfall is the risk measure, the NIG based approach is found to be the clearly preferred method in small samples.

Wilhelmsson (2009) proposed a new model for financial returns based on the NIG distribution with time varying variance, skewness and kurtosis. Bauer (2000) showed symmetric hyperbolic distributions have simple practical VaR computations. Fajardo, Farias and Ornelas (2005) analyzed the use of generalized hyperbolic distributions to VaR calculations with applications in the US Dollar/Brazilian real exchange rate. Through various perspectives, Fajardo, Farias and Ornelas recommended the use of the GH family distribution estimating by the maximum log likelihood method. Huang, et al. (2014) applied the generalized hyperbolic distributions for VaR estimation for the South African mining index. Through the comparison of three subclasses of the generalized hyperbolic distributions using the Akaike information criterion (AIC), the Bayesian information criterion (BIC) and log-likelihoods, Huang, et al. found the generalized hyperbolic (GH) skew Student's $t$ distribution as the most robust model for the South African mining index returns. Finally, Mabitsela, et al. (2015) compared the normal distribution, the Skewed $t$ distribution, the Student's $t$ distribution and the normal inverse Gaussian distribution in quantification of VaR in the South African equity market.

In this paper, we compare the various types of heavy-tailed distribution using the US stock market index returns. In contrast to the literature focusing on the subclasses of the generalized hyperbolic distributions, we consider the generalized hyperbolic distribution itself and allow all of parameters to be estimated. Also, we consider the Skewed $t$ distribution as in Hansen (1994), and our results indicate the Skewed $t$ distribution has superior performance in risk management practice. The remainder of the paper is organized as follows. In Section 2, we introduce the heavy-tailed distributions. Section 3 summarizes the data. The estimation results are in Section 4. Finally, we conclude in Section 5 .

\section{The Heavy-tailed Distributions}

In this section, we introduce four types of heavy-tailed distribution in addition to the normal distribution: (i) the Student's $t$ distribution; (ii) the Skewed $t$ distribution; (iii) the normal reciprocal inverse Gaussian distribution (NRIG); and (iv) the generalized hyperbolic distribution $(\mathrm{GH})$. All the distributions have been standardized to ensure mean and standard deviation equal to zero and one respectively. Their probability density functions are given as follows.

(i) Student's $t$ distribution: 


$$
f\left(e_{t} \mid \psi_{t-1}\right)=\frac{\Gamma\left(\frac{v+1}{2}\right)}{\Gamma\left(\frac{v}{2}\right)[(v-2) \pi]^{1 / 2}}\left(1+\frac{e_{t}^{2}}{(v-2)}\right)^{-\frac{v+1}{2}} .
$$

where $v$ indicates degrees of freedom and $e_{t}$ is daily US equity market index return.

(ii) Skewed $t$ distribution:

$$
f\left(e_{t} \mid v, \beta\right)=\left\{\begin{array}{ll}
b c\left(1+\frac{1}{v-2}\left(\frac{b e_{t}+a}{1-\beta}\right)^{2}\right)^{-(v+1) / 2} & e_{t}<-a / b \\
b c\left(1+\frac{1}{v-2}\left(\frac{b e_{t}+a}{1+\beta}\right)^{2}\right)^{-(v+1) / 2} & e_{t} \geq-a / b
\end{array},\right.
$$

where $e_{t}$ is the standardized $\log$ return, and the constants $a, b$ and $c$ are given by $a=4 \beta c\left(\frac{v-2}{v-1}\right), b^{2}=1+3 \beta^{2}-a^{2}$, and $c=\frac{\Gamma\left(\frac{v+1}{2}\right)}{\sqrt{\pi(v-2)} \Gamma\left(\frac{v}{2}\right)}$. The density function has a mode of $-a / b$, a mean of zero, and a unit variance. The density function is skewed to the right when $\beta>0$, and vice-versa when $\beta<0$. The skewed Student's $t$ distribution specializes to the standard Student's $t$ distribution by setting the parameter $\beta=0$.

The Skewed $t$ distribution is specified as in Hansen (1994). We choose this particular type of Skewed $t$ distribution instead of other forms because the parameters are easy to estimate and its analytical form is close to the standard Student's $t$ distribution, as one can still see the power function form clearly. Meanwhile, it is also commonly used in the finance literature also with good results in our application.

(iii) Normal reciprocal inverse Gaussian distribution (NRIG):

$$
f\left(e_{t} \mid \psi_{t-1}\right)=\frac{\alpha K_{0}\left(\sqrt{\left(\alpha^{2}-1\right)^{2}+\alpha^{2} e_{t}^{2}}\right)}{\pi} \exp \left(\alpha^{2}-1\right) .
$$

where $K_{\lambda}(\cdot)$ is the modified Bessel function of the third kind and index $\lambda=0$ and $\alpha>0$. The NRIG distribution is specified as in Prause (1997). We are particularly interested in the NRIG distribution instead of the NIG distribution, since Guo (2017) show the NRIG distribution has better empirical performance than the NIG distribution under the generalized autoregressive heteroskedasticity $(\mathrm{GARCH})$ model framework.

(iv) Generalized hyperbolic distribution: 


$$
f\left(e_{t} \mid p, b, g\right)=\frac{g^{p}}{\sqrt{2 \pi}\left(b^{2}+g^{2}\right)^{\frac{1}{2}\left(p-\frac{1}{2}\right)} d(p, b, g) K_{p}(g)} q\left(\frac{e_{t}-m(p, b, g)}{d(p, b, g)} ; p, b, g\right)
$$

where $\tilde{R}_{n} \square \frac{K_{n+p}(g)}{g^{n} K_{p}(g)}, \quad d(p, b, g) \square\left[\tilde{R}_{1}+b^{2}\left\{\tilde{R}_{2}-\tilde{R}_{1}^{2}\right\}\right]^{-\frac{1}{2}} \geq 0, \quad$ and $m(p, b, g) \square-b d(p, b, g) \tilde{R}_{1} \cdot p, b$ and $g$ are parameters. The generalized hyperbolic distribution is a standardized version of Prause (1997).

\section{Data}

We fit the heavy tailed distributions with the normalized US equity market index returns. The Standard \& Poor's 500, based on the market capitalizations of 500 large companies having common stock listed on the NYSE or NASDAQ, is considered as one of the best representations of the U.S. stock market. We collected the standardized S\&P 500 daily dividend-adjusted close returns from Yahoo Finance for the period from March 4, 1957 to January 31, 2017, covering all the available data since the index was launched. Figure 1 illustrates the dynamics of S\&P 500, and the biggest spike was observed on October 19, 1987. The recent financial crisis also witnessed significant volatility in the financial market.

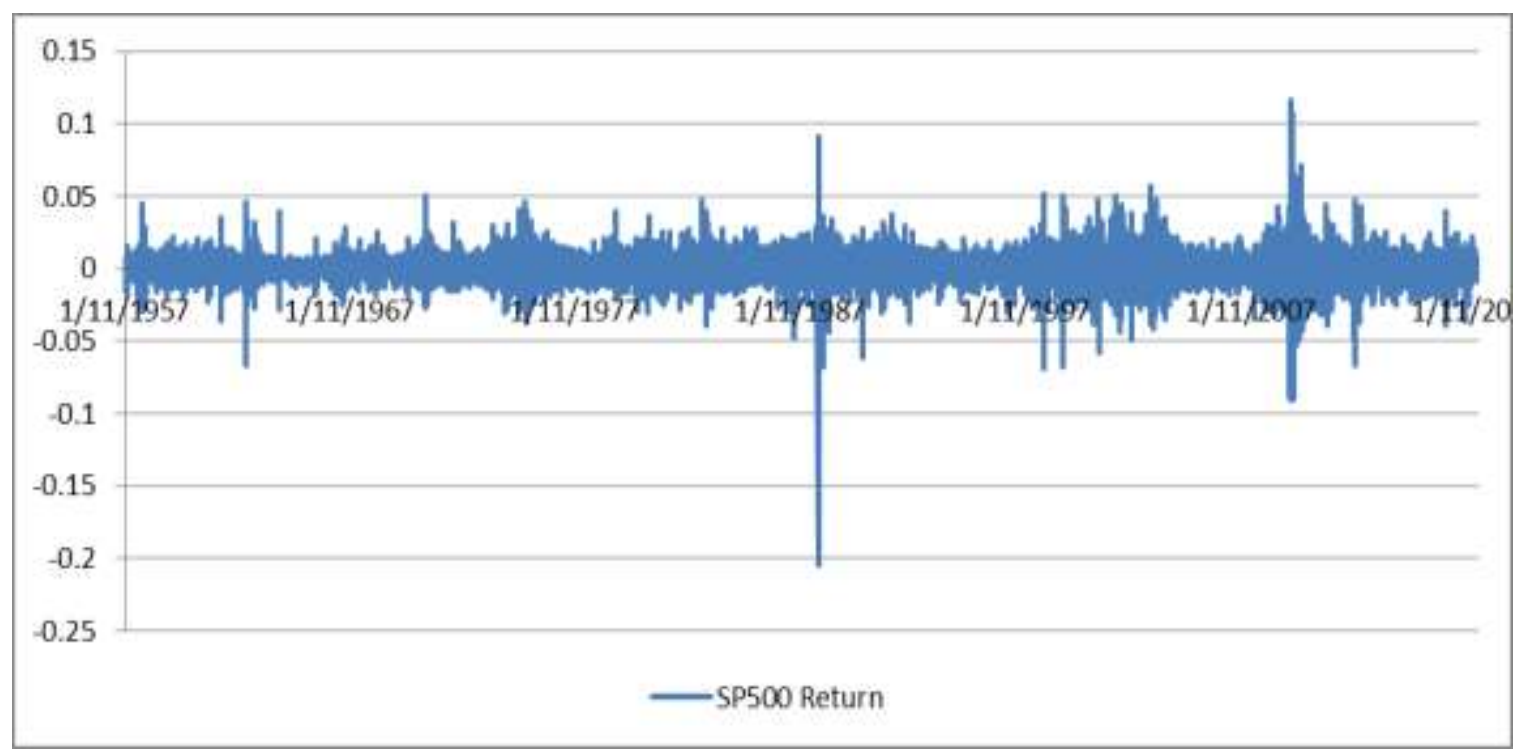

Figure 1: S\&P 500 returns

Table 1 exhibits basic statistics of the S\&P 5 returns. The results show the S\&P 500 daily returns are leptokurtotic and negatively skewed. The extreme downside move is almost twice of the extreme upside move. 
Table 1: Descriptive statistics

\begin{tabular}{|c|c|c|c|c|c|}
\hline $\min$ & $\max$ & mean & std & skewness & Kurtosis \\
\hline \hline$-20.47 \%$ & $11.58 \%$ & $0.03 \%$ & $0.99 \%$ & -0.62 & 20.87 \\
\hline
\end{tabular}

Figure 2 is the histogram of the raw data. We fit the returns by the Gaussian distribution and the Student's $t$ distribution. The upper panel in Figure 2 is fitted by the normal distribution. Clearly, the Student's $t$ distribution has a much better in-sample goodness of fit.
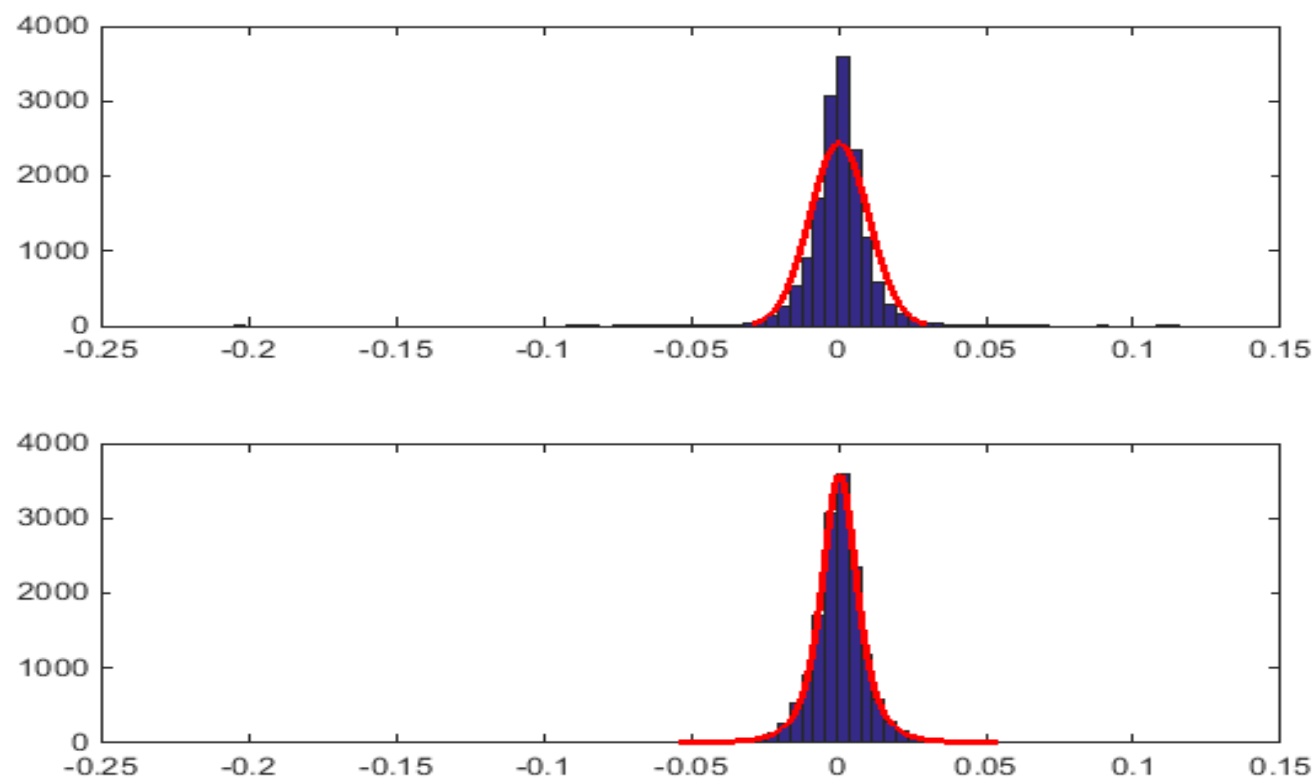

Figure 2: S\&P 500 returns - Normal vs. Student's t

\section{Empirical Results}

\subsection{Parameters Estimation}

We estimated the parameters by the maximum likelihood estimation (MLE) method and the estimation results of the key parameters are given in Table 2. All the parameters are significantly different from zero at $5 \%$ significance level. 
Table 2: Estimated values of key parameters

\begin{tabular}{|l|c|c|c|c|c|}
\hline & Normal & $\begin{array}{c}\text { Student's } \\
\boldsymbol{t}\end{array}$ & Skewed $\boldsymbol{t}$ & NRIG & $\begin{array}{c}\text { Generalized } \\
\text { Hyperbolic }\end{array}$ \\
\hline Symmetric & $\mathrm{Y}$ & $\mathrm{Y}$ & $\mathrm{N}$ & $\mathrm{N}$ & $\mathrm{N}$ \\
\hline Fat-tailed & $\mathrm{N}$ & $\mathrm{Y}$ & $\mathrm{Y}$ & $\mathrm{Y}$ & $\mathrm{Y}$ \\
\hline $\begin{array}{l}\text { Estimated } \\
\text { Parameters }\end{array}$ & & $\begin{array}{c}\mathrm{Nu}= \\
3.86\end{array}$ & $\begin{array}{c}\mathrm{Nu}=3.85 ; \text { beta }=- \\
0.063 \\
(\mathrm{a}=-0.09 ; \mathrm{b}=1.002 ; \\
\mathrm{c}=0.54)\end{array}$ & $\begin{array}{c}\text { alpha } \\
=1.285 ; \\
\text { beta }=-0.097\end{array}$ & $\begin{array}{c}\mathrm{p}=-1.498 ; \\
\mathrm{b}=-0.095 ; \\
\mathrm{g}=0.465\end{array}$ \\
\hline
\end{tabular}

\subsection{Goodness of Fit}

Based on Huber-Carol, et al. (2002) and Taeger and Kuhnt (2014), in this section we compare the four heavy-tailed distributions and the benchmark normal distribution in fitting the S\&P 500 daily returns through four different criteria: (i) Kolmogorov-Smirnov statistic; (ii) Cramer-von Mises criterion; (iii) Anderson-Darling test; and (iv) Akaike information criterion (AIC).

(i) Kolmogorov-Smirnov statistic is defined as the maximum deviation between empirical CDF (cumulative distribution function) $F_{n}(x)$ and tested $\operatorname{CDF} F(x)$ :

$$
D_{n}=\sup _{x}\left|F_{n}(x)-F(x)\right|
$$

where $F_{n}(x)=\frac{1}{n} \sum_{i=1}^{n} I_{[-\infty, x]}\left(X_{i}\right)$.

(ii) Cramer-von Mises criterion is defined as the average squared deviation between empirical CDF and tested CDF:

$$
T=n \int_{-\infty}^{\infty}\left[F_{n}(x)-F(x)\right]^{2} d F(x)=\frac{1}{12 n}+\sum_{i=1}^{n}\left[\frac{2 i-1}{2 n}-F_{n}\left(x_{i}\right)\right]^{2},
$$

(iii) Anderson-Darling test is defined as the weighted-average squared deviation between empirical CDF and tested CDF:

$$
A=n \int_{-\infty}^{\infty} \frac{\left(F_{n}(x)-F(x)\right)^{2}}{F(x)(1-F(x))} d F(x)
$$

and the formula for the test statistic $A$ to assess if data comes from a tested distribution is given by:

$$
A^{2}=-n-\sum_{i=1}^{n} \frac{2 i-1}{n}\left[\ln \left(F\left(x_{i}\right)\right)+\ln \left(1-F\left(x_{i}\right)\right)\right] .
$$

(iv) Akaike information criterion (AIC) is defined as: 


$$
A I C=-2 k-2 \ln (L),
$$

where $L$ is the maximum value of the likelihood function for the model, and $k$ is the number of estimated parameters in the model.

The comparison results are illustrated in Table 3 and Figure 3, indicating the Skewed $t$ distribution has the best goodness of fit compared with other selected types of distribution, followed by the Student's $t$ distribution and the generalized hyperbolic distribution.

Table 3: Comparison of selected types of distribution

\begin{tabular}{|l|c|c|c|c|c|}
\hline & Gaussian & Student's $t$ & Skewed $t$ & NRIG & $\begin{array}{c}\text { Generalized } \\
\text { Hyperbolic }\end{array}$ \\
\hline K-S Test & 0.018 & 0.009 & 0.006 & 0.008 & 0.007 \\
\hline Cv-M Test & 0.025 & 0.016 & 0.013 & 0.019 & 0.015 \\
\hline A-D Test & 1.54 & 1.15 & 1.07 & 1.21 & 1.11 \\
\hline AIC & 61314.5 & 56063.4 & 55949.8 & 56301.6 & 55956.6 \\
\hline
\end{tabular}

\subsection{Value at Risk}

Table 4: Scenarios for SPX shocks

\begin{tabular}{|l|r|r|r|r|r|}
\hline \multicolumn{7}{|c|}{ Left Tail (Daily Loss) } \\
\hline Confidence & $99.999 \%$ & $99.99 \%$ & $99.975 \%$ & $99.95 \%$ & $99.9 \%$ \\
\hline Empirical & $-22.23 \%$ & $-14.75 \%$ & $-8.87 \%$ & $-6.83 \%$ & $-5.96 \%$ \\
\hline Normal & $-4.22 \%$ & $-3.68 \%$ & $-3.44 \%$ & $-3.25 \%$ & $-3.06 \%$ \\
\hline T & $-13.89 \%$ & $-11.02 \%$ & $-8.27 \%$ & $-6.09 \%$ & $-5.05 \%$ \\
\hline Skewed T & $-15.80 \%$ & $-12.07 \%$ & $-8.88 \%$ & $-6.50 \%$ & $-5.39 \%$ \\
\hline NIG & $-10.50 \%$ & $-8.45 \%$ & $-6.58 \%$ & $-5.10 \%$ & $-4.36 \%$ \\
\hline GH & $-12.21 \%$ & $-9.47 \%$ & $-7.20 \%$ & $-5.50 \%$ & $-4.66 \%$ \\
\hline \multicolumn{5}{|c|}{ Right Tail (Daily Loss) } \\
\hline Confidence & $0.001 \%$ & $0.01 \%$ & $0.025 \%$ & $0.05 \%$ & $0.1 \%$ \\
\hline Empirical & $13.23 \%$ & $11.18 \%$ & $8.09 \%$ & $6.35 \%$ & $5.06 \%$ \\
\hline Normal & $4.22 \%$ & $3.68 \%$ & $3.44 \%$ & $3.25 \%$ & $3.06 \%$ \\
\hline T & $13.89 \%$ & $11.02 \%$ & $8.27 \%$ & $6.09 \%$ & $5.05 \%$ \\
\hline Skewed T & $12.26 \%$ & $10.19 \%$ & $7.83 \%$ & $5.81 \%$ & $4.83 \%$ \\
\hline NIG & $10.50 \%$ & $8.45 \%$ & $6.58 \%$ & $5.10 \%$ & $4.36 \%$ \\
\hline GH & $9.77 \%$ & $8.17 \%$ & $6.47 \%$ & $5.01 \%$ & $4.28 \%$ \\
\hline
\end{tabular}

In financial mathematics and financial risk management, $\mathrm{VaR}$ is defined as: for a given position, time horizon, and probability $\mathrm{p}$, the $\mathrm{p}$ VaR is defined as a threshold loss value, 
such that the probability that the loss on the position over the given time horizon exceeds this value is $\mathrm{p}$. with the estimated parameters in Section 4.1, we calculate VaRs for different confidence levels:

$$
\operatorname{VaR}_{\alpha}\left(e_{t}\right)=\inf \left\{e \in \square: P\left(e_{t}>e\right) \leq 1-\alpha\right\},
$$

where $\alpha \in(0,1)$ is the confidence level. We select the following levels for downside moves: $\{99.999 \%, 99.99 \%, 99.975 \%, 99.95 \%, 99.9 \%\}$, and for upside moves: $\{0.001 \%$, $0.01 \%, 0.025 \%, 0.05 \%, 0.1 \%\}$. From Equation (9), the VaR levels are given as in Table 4. Table 4 indicates that the Skewed $t$ distribution has the closest VaRs to the nonparametric historical VaRs compared with other types of distributions.

We further draw the tail parts of the distributions in Figure 3. Again, the figure illustrates that the Skewed $t$ distribution has best goodness-of-fit in the tail parts, followed by the Student's $t$ distribution and the generalized hyperbolic distribution. The normal distribution has almost no predictive ability for the tail parts.
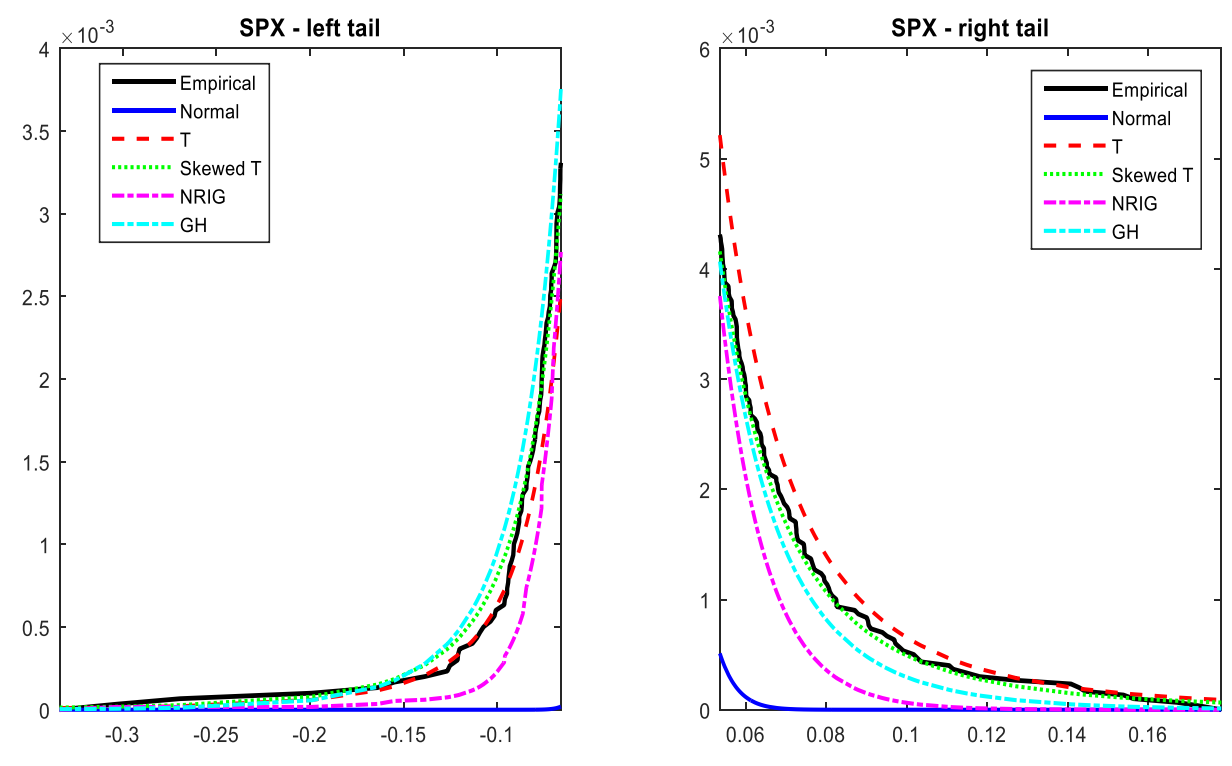

Figure 3: Comparison of selected types of distribution - tails

\section{Conclusions}

Value-at-Risk (VaR), defined as the worst expected loss over a given period at a specified confidence level, has become one of the most widely used risk measures by financial institutions and regulatory. When evaluating $\mathrm{VaR}$ for financial assets the distribution of the returns of the underlying asset play an important role. The methods to estimate VaR can be classified into two groups, i.e., the parametric VaR and non-parametric VaR. Parametric VaR assumes that financial returns following a specific statistical distribution 
(e.g., Gaussian and Student $t$ distribution). The quality of parametric VaR is therefore dependent on how well the statistical distribution captures the asymmetric and leptokurtic behavior of the financial returns. A more reliable statistical distribution could result in more trustworthy estimations of risk and lead to improved risk management practice.

In this paper, we consider four widely used heavy-tailed distributions, and fit them with the S\&P 500 returns. Through a variety of criteria, we found the Skewed $t$ distribution in Hansen (1994) has the best in-sample goodness of fit compared with the Student's $t$ distribution, the normal reciprocal inverse Gaussian distribution and the generalized hyperbolic distribution. Moreover, we showed the Skewed $t$ distribution could generate Value at Risk estimates closest to the nonparametric historical Value at Risk estimates. For the future research, one may consider combining the volatility clustering effect with the heavy tails phenomenon as in Guo (2017) and investigate their implications in risk measure estimates.

\section{References}

Akaike, H. (1973), "Information theory and an extension of the maximum likelihood principle", in Petrov, B.N.; Csáki, F., 2nd International Symposium on Information Theory, Tsahkadsor, Armenia, USSR, pp. 267-281.

Barndorff-Nielsen, O.(1977), "Exponentially decreasing distributions for the logarithm of particle size," Proceedings of the Royal Society, vol. 353, pp. 401-419.

Barndorff-Nielsen, O.(1997), "Normal inverse Gaussian distributions and stochastic volatility modeling," Scandinavian Journal of Statistics, vol. 24, pp.1-13.

Bauer, C. (2000), "Value at Risk using hyperbolic distributions," Journal of Economics and Business, vol. 52, pp. 455-467.

Cont, R. (2001), "Empirical properties of asset returns: stylized facts and statistical issues," Quantitative Finance, vol. 223-236.

Dokov, J., S. Stoyanov and S. Rachev (2008), "Computing VaR and AVaR of skewed-T distribution,” Journal of Applied Functional Analysis, vol. 3, pp. 189-207.

Duffie, D. and J. Pan (1997), “An overview of Value at Risk,” Journal of Derivatives, vol. 4, pp. 7-49.

Fajardo, J., A. Farias and J. Ornelas (2005), “Analyzing the use of generalized hyperbolic distributions to Value at Risk calculations," Brazilian Journal of Applied Economics, vol. 9, pp. 25-38.

Figueroa-Lopez, J., S. Lancette, K. Lee and Y. Mi (2011), "Estimation of NIG and VG models for high frequency financial data," in Handbook of Modeling HighFrequency Data in Finance, edited by F. G. Viens, M.C. Mariani and I. Florescu, John Wiley \& Sons, Inc., USA.

Guo, Z. (2017), "Empirical performance of GARCH models with heavy-tailed innovations," Wells Fargo Securities, working paper.

Huber-Carol, C., N. Balakrishnan, M. Nikulin and M. Mesbah (2002), Goodness-of-Fit Tests and Model Validity, Springer.

Hansen, B. (1994), "Autoregressive conditional density estimation," International Economic Review, vol. 35, pp. 705-730. 
Huang, C., C. Knowledge, C. Huang and H. Jahvaid (2014), "Generalized hyperbolic distributions and Value-At-Risk estimation for the South African mining index," International Business \& Economics Research Journal, vol. 13, pp. 319-332.

Mabitsela, L., E. Mare and R. Kufakunesu (2015), "Quantification of VaR: a note on VaR valuation in the South African equity market," Journal of Risk and Financial Management, vol. 8, pp. 103-126.

Mandelbrot, B. (1963), "New methods in statistical economics," Journal of Political Economy, vol. 71, pp. 421-440.

Prause, K. (1999), "The generalized hyperbolic model: estimation, financial derivatives, and risk measures," Ph.D. Dissertation.

Rradley, O. and M. Taqqu (2003), "Financial risk and heavy tails," Handbook of Heavy Tailed Distributions in Finance, edited by S. Rachev, Elsevier.

Socgnia, V. and D. Wilcox (2014), "A comparison of generalized hyperbolic distribution models for equity returns," Journal of Applied Mathematics, vol. 2014, pp. 23-38.

Taeger, D. and S. Kuhnt (2014), "Goodness-of-fit tests," Statistical Hypothesis Testing with SAS and R, Wiley Online Library.

Venter, J. and P. de Jongh (2002), "Risk estimation using the normal inverse Gaussian distribution," Journal of Risk, vol. 4, pp.1-24.

Wilhelmsson, A. (2009), "Value at Risk with time varying variance, skewness and kurtosis - the NIG-ACD model," Econometrics Journal, vol. 12, pp. 82-104.

Zhu, D. and J. Galbraith (2012), “A generalized asymmetric Student-t distribution with application to financial econometrics," Journal of Econometrics, vol. 157, pp. 297305. 